\title{
Synthesis and Characterization of N-Substituted Tetrahydroiso- quinoline Derivatives via a Pictet-Spengler Condensation
}

\author{
Heta A. Patel and Arun L. Patel ${ }^{*}$
}

Department of Chemistry, Faculty of Science, The Maharaja Sayajirao University of Baroda, Vadodara-390 002, India

\begin{abstract}
Synthesis of $\mathrm{N}$-substituted 1,2,3,4 -tetrahydroisoquinoline derivatives and bis-isoquinoline has been carried out via a Pictet-Spengler condensation. Tetrahydroisoquinolines were obtained from 2-(3',4'-dimethoxyphenyl) ethylamine in four steps. The entire synthesized compounds were characterized by IR, ${ }^{1} \mathrm{H}$ NMR and mass spectral data.
\end{abstract}

Keywords: Tetrahydroisoquinoline, Pictet-Spengler reaction, Imine, Trifluoroacetic acid.

\section{INTRODUCTION}

Tetrahydroisoquinoline (THIQ) alkaloids have attracted considerable interest over the years due to their potent biological activities. A wide range of $\mathrm{N}$ substituted 1,2,3,4 -tetrahydroisoquinolines has been found to be a useful starting material for the construction of a variety of medically attractive intermediates $[1,2]$. THIQ derivatives possess neuroprotective activity against neurological diseases such as epilepsy, ischemia, Parkinson's disease and multiple sclerosis [3-6]. The natural alkaloids containing THIQ unit are generally optically active compounds and possess antihypertensive, hemostatic, smooth or skeletal muscle relaxant, antispasmodic, antitussive, antimalarial, narcotic, analgesic or antipyretic activities [7-10]. Moreover, THIQ is a basic structural unit of many alkaloids isolated from natural sources which showed antitumor ${ }^{11}$, antimicrobial ${ }^{12}$ and other biological activities [13-17]. The Pictet-Spengler reaction and Bischler-Napieralski reaction are the key reactions for construction of the isoquinoline skeleton [18]. The Pictet-Spengler reaction is an acid-catalyzed intramolecular cyclization of the intermediate imine, which is formed by condensation of 2-arylethylamine with a carbonyl compound (Scheme 1) [19-21].

\section{Pictet-Spengler Reaction}

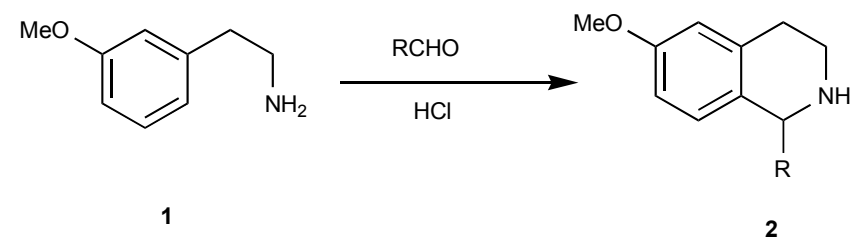

Scheme 1:

*Address correspondence to this author at the Department of Chemistry, Faculty of Science, The Maharaja Sayajirao University of Baroda, Vadodara390 002, India; Tel: +91-265-2795552; Fax: +91-265-2792277;

E-mail: arunpatel_5376@yahoo.co.in
Pictet-Spengler reaction was used in cyclocondensation of $\beta$-phenethylamine (3) with formaldehyde dimethyl acetal (4) in the presence of hydrochloric acid to give 1-methyl-1,2,3,4- tetrahydroisoquinoline (5) [22]. The reaction was later modified and subsequently intramolecular electrophilic substitution was proposed as depicted in (Scheme 2) [23].

To improve the activity of THIQ based derivatives for various biological purposes such as antispasmodic, anticonvulsant efficacy, antimalarial, antimicrobial etc., we have introduced an extra heterocyclic ring in it. It is expected that such modification can improve the potential of resulted compounds. Therefore, we are reporting a four step synthetic strategy that gives $\mathrm{N}$ substituted tetrahydro-isoquinoline derivatives. In this work we have also reported bis-isoquinoline synthesized by condensation of 2-(3',4'dimethoxyphenyl)ethylamine and pthalal-dehyde.

\section{RESULTS AND DISCUSSION}

Scheme 3 shows the synthetic route of six new THIQ derivatives. We have also synthesized novel bisTHIQ (16) by adopting similar synthetic methodology (Scheme 4).

First step of the synthesis is the condensation of 2(3',4'-dimethoxyphenyl)ethylamine and substituted aldehydes in anhydrous toluene. It is followed by cyclization in presence of trifluoroacetic acid (TFA) to afford 1-(4-substitutedphenyl)-1,2,3,4-tetrahydro-6,7dimethoxyisoquinoline. Further, product of the second step was treated with chloroacetyl chloride afforded the expected product in a good yield. The synthesis of proposed isoquinoline derivative has been completed by treating 2-chloro-1-(3,4-dihydro-6,7-dimethoxy-1-(4substitutedphenyl)isoquinolin-2(1H)-yl)ethanone with morpholine/N-methylpiperazine in presence of activated $\mathrm{K}_{2} \mathrm{CO}_{3}$. 


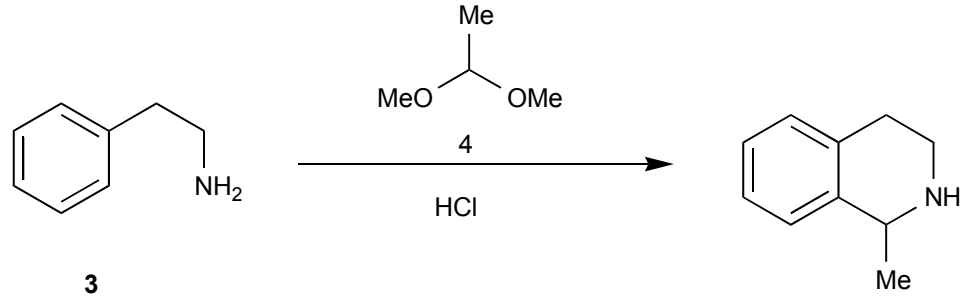

5<smiles>NCCc1ccccc1</smiles>

3<smiles>[R]C[N+]1([2H])CCc2ccccc2C1</smiles>

6

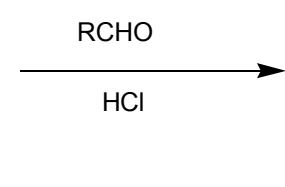<smiles>[R]C1NCCc2ccccc21</smiles>

7

Scheme 2:<smiles>[R]c1ccc(C=O)cc1</smiles><smiles>[X]CCN(CO)CC(=O)N1CCc2cc(OC)c(OC)cc2C1c1ccc([R])cc1</smiles>

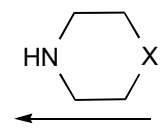<smiles>[R]c1ccc(/C=N/CCc2ccc(OC)c(OC)c2)cc1</smiles>

10a-c<smiles>[R]c1ccc(C2NCCc3cc(OC)c(OC)cc32)cc1</smiles>

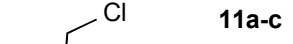<smiles>O=C(Cl)C1CCCC1</smiles>
$\mathrm{C}^{\mathrm{C}^{3}}$ 


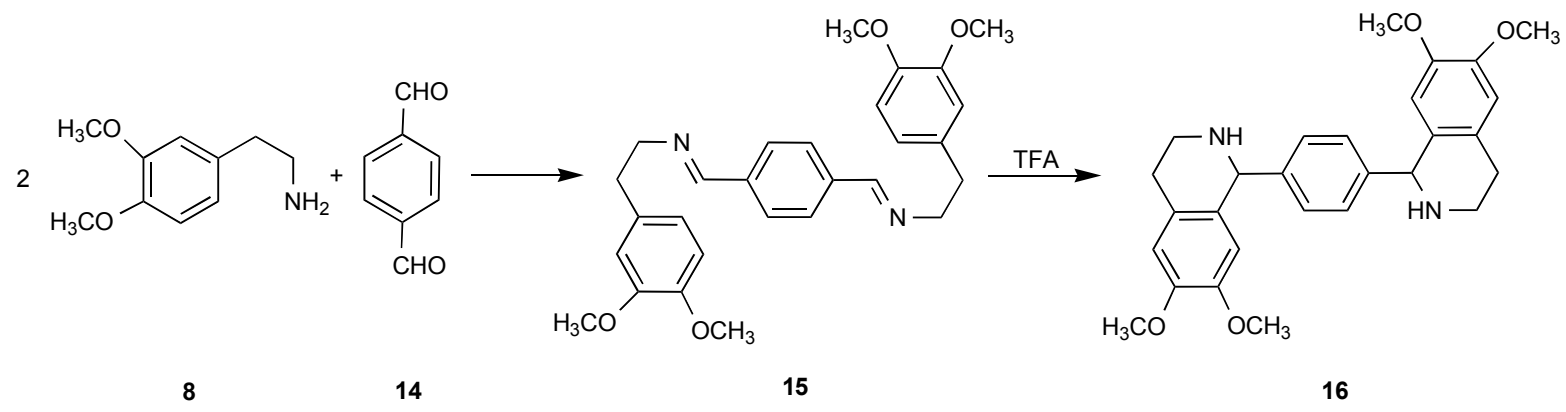

Scheme 4:

were recorded on a Shimadzu Prestige 21 spectrometer. Melting points were recorded in a Thiele's tube using paraffin oil and are uncorrected.

General Procedure for the Synthesis of N-(Substitutedbenzylidene)-2-(3,4-dimethoxyphenyl) ethanamine $(10 \mathrm{a}-\mathrm{c})$

A mixture of 2-(3',4'-dimethoxyphenyl)ethylamine $(1.8 \mathrm{~g}, 10 \mathrm{mmol})$ and suitable aldehyde derivative $(12 \mathrm{mmol})$ in anhydrous toluene $(50 \mathrm{~mL})$ was refluxed for $3 \mathrm{~h}$ and then cooled and evaporated in vacuo. The oil residue was treated with diethyl ether to give a solid residue, which was crystallized from ethanol to afford the desired imine.

\section{(E)-N-benzylidene-2-(3,4-dimethoxyphenyl) ethanamine (10a)}

Yield $71.1 \%$, Pale Yellow crystals. Melting point 119-121 ${ }^{\circ} \mathrm{C} .{ }^{1} \mathrm{H}$ NMR (400 MHz, $\left.\mathrm{CDCl}_{3}\right): \delta$ 2.92-3.95 (t, $2 \mathrm{H}, \mathrm{ArCH}_{2}$ ), 3.79 (s, 3H, OMe), 3.82 (s, 3H, OMe), 3.80-3.83 (m, 2H, NCH 2$), 6.58-6.67$ (m, 3H, ArH), 7.42$7.46(\mathrm{~m}, 3 \mathrm{H}, \mathrm{ArH}), 7.63-7.66(\mathrm{~m}, 2 \mathrm{H}, \mathrm{ArH}), 8.16(\mathrm{~s}, 1 \mathrm{H}$, $=\mathrm{CH}$ ). Mass (EI): m/z (\%): $\mathrm{M}^{+} 269$ (10.0), 270 (18.47), 192 (100). IR (KBr, cm $\left.{ }^{-1}\right): 1588$.

\section{(E)-N-(4-nitrobenzylidene)-2-(3,4-dimethoxyphenyl) ethanamine (10b)}

Yield $74.3 \%$, Yellow crystals. Melting point $130{ }^{\circ} \mathrm{C}$. ${ }^{1} \mathrm{H}$ NMR (400 MHz, $\mathrm{CDCl}_{3}$ ): $\delta$ 2.98-3.01 (t, 2H, $\mathrm{ArCH}_{2}$ ), 3.82 (s, 3H, OMe), 3.85 (s, 3H, OMe), 3.89-3.93 (t, 2H, $\left.\mathrm{NCH}_{2}\right), 6.73-6.81(\mathrm{~m}, 3 \mathrm{H}, \mathrm{ArH}), 7.85-7.87$ (d, 2H, ArH), $8.19(\mathrm{~s}, 1 \mathrm{H},=\mathrm{CH}), 8.25-8.27(\mathrm{~d}, 2 \mathrm{H}, \mathrm{ArH})$. Mass $(\mathrm{El})$ : $\mathrm{m} / \mathrm{z}(\%): \mathrm{M}^{+} 313$ (100), 150 (58.47), 163 (18.42), 117 (28.92), 77 (15.21). IR ( $\left.\mathrm{KBr}, \mathrm{cm}^{-1}\right): 1597$.

\section{(E)-N-(4-chlorobenzylidene)-2-(3,4-dimethoxy- phenyl)ethanamine (10c)}

Yield $77.1 \%$, Pale Yellow Crystals. Melting point 99-102 ${ }^{\circ} \mathrm{C} .{ }^{1} \mathrm{H}$ NMR $\left(400 \mathrm{MHz}, \mathrm{CDCl}_{3}\right): \delta$ 2.95-2.98 (t, $2 \mathrm{H}, \mathrm{ArCH}_{2}$ ), 3.81 (s, 3H, OMe), 3.82-3.85 (m, 2H, $\left.\mathrm{NCH}_{2}\right), 3.86(\mathrm{~s}, 3 \mathrm{H}, \mathrm{OMe}), 6.74-6.81(\mathrm{~m}, 3 \mathrm{H}, \mathrm{ArH})$,
7.38-7.40 (d, 2H, ArH), 7.63-7.66 (d, 2H, ArH), 8.09 (s, $1 \mathrm{H},=\mathrm{CH})$. Mass (El): $\mathrm{m} / \mathrm{z}(\%): \mathrm{M}^{+}$302.5(11.72), 150(100), 152.5(27.67), 124.5(24.92), 89(15.12). IR $\left(\mathrm{KBr}, \mathrm{cm}^{-1}\right): 1571$.

General Procedure for the Synthesis of 1-(4-Substitutedphenyl)-1,2,3,4-tetrahydro-6,7-dimethoxyisoquinoline (11a-c)

TFA (10mL) was added to a solution of benziliden [2-(3,4-dimethoxyphenyl)ethyl]amine (3.2mmol), and the mixture was refluxed for $1.5 \mathrm{~h}$. The reaction was quenched by adding water, and the mixture was basified ( $\mathrm{pH} 8-9)$ with sodium hydroxide to give the isoquinoline derivative as a solid. The crude product was collected by filtration and purified by crystallization with $\mathrm{MeOH}$ to afford compounds.

\section{1,2,3,4-tetrahydro-6,7-dimethoxy-1-phenylisoquino- line (11a)}

Yield 68.3\%, White crystals. Melting point 110-111 ${ }^{\circ} \mathrm{C} .{ }^{1} \mathrm{H}$ NMR $\left(400 \mathrm{MHz} \mathrm{CDCl}_{3}\right): \delta 1.95(\mathrm{~s}, 1 \mathrm{H}, \mathrm{NH})$, 2.65-2.78 (m, 2H, $\left.\operatorname{ArCH}_{2}\right), 2.86-3.10\left(\mathrm{~m}, 2 \mathrm{H}, \mathrm{NCH}_{2}\right)$, 3.69 (s, 3H, OMe), 3.77 (s, 3H, OMe), 5.20 (s, 1H, CH), 6.36 (s, 2H, ArH ), 6.87 (s, 1H, ArH), 7.23-7.39 (d, 2H, $\mathrm{ArH}), 7.88-7.96(\mathrm{~d}, 2 \mathrm{H}, \mathrm{ArH})$. Mass (El): $\mathrm{m} / \mathrm{z}(\%): \mathrm{M}^{+}$ 269 (42.0), 268 (45), 192 (100). IR ( $\left.\mathrm{KBr}, \mathrm{cm}^{-1}\right): 3434$.

\section{1,2,3,4-tetrahydro-6,7-dimethoxy-1-(4-nitrophenyl) isoquinoline (11b)}

Yield $65.0 \%$, White crystals. Melting point $144{ }^{\circ} \mathrm{C}$. ${ }^{1} \mathrm{H}$ NMR $\left(400 \mathrm{MHz}, \mathrm{CDCl}_{3}\right): \delta 1.99$ (s, 1H, NH), 2.75$2.98\left(\mathrm{~m}, 2 \mathrm{H}, \mathrm{ArCH}_{2}\right), 3.04-3.20\left(\mathrm{~m}, 2 \mathrm{H}, \mathrm{NCH}_{2}\right), 3.65$ (s, $3 \mathrm{H}, \mathrm{OMe}$ ), 3.89 (s, 3H, OMe), 5.16 (s, 1H, CH), 6.16 (s, $1 \mathrm{H}, \mathrm{ArH}), 6.67$ (s, 1H, ArH), 7.45-7.47 (d, 2H, ArH), 8.18-8.20 (d, 2H, ArH). Mass (El): m/z (\%): $\mathrm{M}^{+} 314$ (20.71), 312 (100), 192 (64.53). IR ( $\left.\mathrm{KBr}, \mathrm{cm}^{-1}\right): 3436$.

\section{1-(4-chlorophenyl)-1,2,3,4-tetrahydro-6,7-dimetho- xyisoquinoline (11c)}

Yield $70.1 \%$, White crystals. Melting point $106{ }^{\circ} \mathrm{C}$. ${ }^{1} \mathrm{H}$ NMR (400 MHz, $\mathrm{CDCl}_{3} \delta 2.19$ (s, 1H, NH), 2.79- 
$3.24\left(\mathrm{~m}, 4 \mathrm{H}, \mathrm{ArCH}_{2} \mathrm{CH}_{2}\right), 3.67$ (s, 3H, OMe), 3.89 (s, $3 \mathrm{H}, \mathrm{OMe}), 5.10(\mathrm{~s}, 1 \mathrm{H}, \mathrm{CH}), 6.21(\mathrm{~s}, 1 \mathrm{H}, \mathrm{ArH}), 6.70(\mathrm{~s}$, $1 \mathrm{H}, \mathrm{ArH}), 7.18-7.25(\mathrm{~d}, 2 \mathrm{H}, \mathrm{ArH}), 7.29-7.33(\mathrm{~d}, 2 \mathrm{H}$, $\mathrm{ArH})$. Mass (El): $\mathrm{m} / \mathrm{z}(\%): \mathrm{M}^{+} 303.5(44.79), 302.5$ (73.95), 192 (100). IR (KBr, cm $\left.{ }^{-1}\right): 3436$.

General Procedure for the Synthesis of 2-chloro-1(3,4-dihydro-6,7-dimethoxy-1-(4-substitutedphenyl) isoquinolin-2(1H)-yl)ethanone (12a-c)

To a solution of 1-phenyl-6,7-dimethoxy-1,2,3,4tetrahydroisoquinolines $(1.5 \mathrm{mmol})$ and triethylamine $(2.19 \mathrm{mmol})$ in dry dichloromethane $(5 \mathrm{ml})$, chloroacetylchloride $(1.7 \mathrm{mmol}))$ was added slowly. During addition the reaction mixture was kept in ice. On addition the mixture was stirred at room temperature for $4 \mathrm{~h}$. After the completion of the reaction, evaporated off the solvent, diluted the residue with water and extracted with ethylacetate. The collective organic portion was washed with brine and dried over $\mathrm{Na}_{2} \mathrm{SO}_{4}$. It was finally concentrated and chromatographed on silica gel using ethylacetate-petroleum ether as eluent.

\section{2-chloro-1-(3,4-dihydro-6,7-dimethoxy-1-phenyliso- quinolin-2(1H)-yl)ethanone (12a)}

Yield $54.5 \%$, White powder. Melting point 219-220 ${ }^{\circ} \mathrm{C} .{ }^{1} \mathrm{H}$ NMR $\left(400 \mathrm{MHz}, \mathrm{CDCl}_{3}\right): \delta$ 2.86-2.98 (m, $2 \mathrm{H}$, $\left.\mathrm{ArCH}_{2}\right), 3.40-3.47\left(\mathrm{~m}, 2 \mathrm{H}, \mathrm{NCH}_{2}\right), 3.72(\mathrm{~s}, 3 \mathrm{H}, \mathrm{OMe})$, $3.31(\mathrm{~s}, 3 \mathrm{H}, \mathrm{OMe}), 4.22\left(\mathrm{~s}, 2 \mathrm{H}, \mathrm{COCH}_{2} \mathrm{Cl}\right), 6.42(\mathrm{~s}, 1 \mathrm{H}$, $\mathrm{CH}), 6.66(\mathrm{~s}, 1 \mathrm{H}, \mathrm{ArH}), 6.73(\mathrm{~s}, 1 \mathrm{H}, \mathrm{ArH}), 7.38-7.41$ (d, $2 \mathrm{H}, \mathrm{ArH}), 8.10-8.13$ (d, $2 \mathrm{H}, \mathrm{ArH}$ ). Mass (El): $\mathrm{m} / \mathrm{z}(\%)$ : $\mathrm{M}^{+} 345.5$ (12.11), 310.0 (100). IR (KBr, $\left.\mathrm{cm}^{-1}\right): 1646$.

\section{2-chloro-1-(3,4-dihydro-6,7-dimethoxy-1-(4-nitro- phenyl) isoquinolin-2(1H)-yl)ethanone (12b)}

Yield $58.1 \%$, White powder. Melting point $172{ }^{\circ} \mathrm{C}$. ${ }^{1} \mathrm{H}$ NMR $\left(400 \mathrm{MHz}, \mathrm{CDCl}_{3}\right): \delta 2.80-3.16(\mathrm{~m}, 2 \mathrm{H}$, $\left.\mathrm{ArCH}_{2}\right), 3.38-3.46\left(\mathrm{~m}, 2 \mathrm{H}, \mathrm{NCH}_{2}\right), 3.70(\mathrm{~s}, 3 \mathrm{H}, \mathrm{OMe})$, $3.91(\mathrm{~s}, 3 \mathrm{H}, \mathrm{OMe}), 4.17\left(\mathrm{~s}, 2 \mathrm{H}, \mathrm{COCH}_{2} \mathrm{Cl}\right), 6.47(\mathrm{~s}, 1 \mathrm{H}$, $\mathrm{CH}), 6.71$ (s, 1H , ArH), 6.83 (s, 1H, ArH), 7.43-7.45 (d, $2 \mathrm{H}, \mathrm{ArH}), 8.13-8.15$ (d, $2 \mathrm{H}, \mathrm{ArH})$. Mass (El): $\mathrm{m} / \mathrm{z}(\%)$ : $\mathrm{M}^{+} 390.5$ (68.91), 354.5 (100), 268.5 (18.06), 76 (14.42). IR (KBr, $\left.\mathrm{cm}^{-1}\right): 1637$.

\section{2-chloro-1-(1-(4-chlorophenyl)-3,4-dihydro-6,7-di- methoxyisoquinolin-2(1H)-yl)ethanone (12c)}

Yield $55.9 \%$, White powder. Melting point $148{ }^{\circ} \mathrm{C}$. ${ }^{1} \mathrm{H}$ NMR $\left(400 \mathrm{MHz}, \mathrm{CDCl}_{3}\right): \delta$ 2.76-2.96 (m, 2H, $\left.\mathrm{ArCH}_{2}\right), 3.42-3.56\left(\mathrm{~m}, 2 \mathrm{H}, \mathrm{NCH}_{2}\right), 3.71$ (s, 3H, OMe), $3.87(\mathrm{~s}, 3 \mathrm{H}, \mathrm{OMe}), 4.23\left(\mathrm{~s}, 2 \mathrm{H}, \mathrm{COCH}_{2} \mathrm{Cl}\right), 6.66(\mathrm{~s}, 1 \mathrm{H}$, $\mathrm{CH}), 6.78(\mathrm{~s}, 1 \mathrm{H}, \mathrm{ArH}), 6.91(\mathrm{~s}, 1 \mathrm{H}, \mathrm{ArH}), 7.52-7.58(\mathrm{~d}$, $2 \mathrm{H}, \mathrm{ArH}), 8.23-8.26$ (d, $2 \mathrm{H}, \mathrm{ArH})$. Mass (El): $\mathrm{m} / \mathrm{z}(\%)$ : $\mathrm{M}^{+} 380$ (5.0), 344.5 (76.35), 343 (100), 302.5 (6.14). IR $\left(\mathrm{KBr}, \mathrm{cm}^{-1}\right): 1651$.
Procedure for the Synthesis of 1-(3,4-dihydro-6,7dimethoxy-1-(4-nitrophenyl)isoquinolin-2(1H)-yl)-2(4-methylpiperazin-1-yl)ethanone (13a)

A solution of $\mathrm{N}$ - methyl piperizine $(0.063 \mathrm{~g}$, $0.63 \mathrm{mmol})$ in DMF $(1.5 \mathrm{ml})$ was added slowly to a solution of $12 \mathrm{a}(0.21 \mathrm{~g}, 0.6 \mathrm{mmol})$ and activated $\mathrm{K}_{2} \mathrm{CO}_{3}$ $(0.25 \mathrm{~g}, 1.83 \mathrm{mmol})$ in DMF $(2 \mathrm{ml})$. The mixture was stirred for $3 \mathrm{~h}$ at $60^{\circ} \mathrm{C}$. After the completion of reaction, diluted the reaction mixture with water and extracted with ethylacetate. The collective organic portion was washed with brine and dried over $\mathrm{Na}_{2} \mathrm{SO}_{4}$. It was finally concentrated and chromatographed on silica gel using ethylacetate-petroleum ether as eluent to obtain desired compound in as a gummy material. Compounds $13 \mathrm{~b}-\mathrm{f}$ also prepared by similar experimental conditions.

\section{1-(3,4-dihydro-6,7-dimethoxy-1-phenylisoquinolin- 2(1H)-yl)-2-(4-methylpiperazin-1-yl)ethanone (13a)}

Yield $53.2 \%$, Gummy material. ${ }^{1} \mathrm{H}$ NMR $(400 \mathrm{MHz}$, $\left.\mathrm{CDCl}_{3}\right): \delta 2.24\left(\mathrm{~s}, 3 \mathrm{H}, \mathrm{NCH}_{3}\right), 2.36-2.43(\mathrm{~m}, 8 \mathrm{H}$, piperazine $), 2.76-2.82\left(\mathrm{~m}, 2 \mathrm{H}, \mathrm{ArCH}_{2}\right), 3.12(\mathrm{~S}, 2 \mathrm{H}$, $\left.\mathrm{COCH}_{2} \mathrm{~N}\right) 3.42-3.50\left(\mathrm{~m}, 2 \mathrm{H}, \mathrm{NCH}_{2}\right), 3.69(\mathrm{~s}, 3 \mathrm{H}, \mathrm{OMe})$, 3.75 (s, 3H, OMe), 6.28 (s, 1H, CH), 6.56 (s, 1H ,ArH), 6.77 (s, $1 \mathrm{H}, \mathrm{ArH}), 7.46-7.54(\mathrm{~m}, 5 \mathrm{H}, \mathrm{ArH})$. Mass (El): m/z (\%): $\mathrm{M}^{+} 409$ (8.43), 267 (4.00), 113 (100). IR (KBr, $\left.\mathrm{cm}^{-1}\right): 1637$.

\section{1-(3,4-dihydro-6,7-dimethoxy-1-phenylisoquinolin- 2(1H)-yl)-2-morpholinoethanone (13b)}

Yield $51.6 \%$, Gummy material. ${ }^{1} \mathrm{H}$ NMR $(400 \mathrm{MHz}$, $\left.\mathrm{CDCl}_{3}\right): \delta 2.40-2.48\left(\mathrm{~m}, 4 \mathrm{H}, \mathrm{N}\left(\mathrm{CH}_{2}\right)_{2}\right), 2.71-2.78(\mathrm{~m}$, $\left.4 \mathrm{H}, \mathrm{O}\left(\mathrm{CH}_{2}\right)_{2}\right), 2.78-2.84\left(\mathrm{~m}, 2 \mathrm{H}, \mathrm{ArCH}_{2}\right), 3.28(\mathrm{~S}, 2 \mathrm{H}$, $\left.\mathrm{COCH}_{2} \mathrm{~N}\right), 3.44-3.54\left(\mathrm{~m}, 2 \mathrm{H}, \mathrm{NCH}_{2}\right), 3.71(\mathrm{~s}, 3 \mathrm{H}$, $\mathrm{OMe}), 3.79(\mathrm{~s}, 3 \mathrm{H}, \mathrm{OMe}), 6.24(\mathrm{~s}, 1 \mathrm{H}, \mathrm{CH}), 6.66(\mathrm{~s}, 1 \mathrm{H}$ ,ArH), $6.69(\mathrm{~s}, 1 \mathrm{H}, \mathrm{ArH}), 7.38-7.52(\mathrm{~m}, 5 \mathrm{H}, \mathrm{ArH})$. Mass (EI): m/z (\%): M+ 396 (10.13), 267 (6.00), 100 (100). IR $\left(\mathrm{KBr}, \mathrm{cm}^{-1}\right): 1633$.

\section{1-(3,4-dihydro-6,7-dimethoxy-1-(4-nitrophenyl)iso- quinolin-2(1H)-yl)-2-(4-methylpiperazin-1- yl)ethanone (13c)}

Yield $55.0 \%$, Gummy material. ${ }^{1} \mathrm{H}$ NMR $(400 \mathrm{MHz}$, $\left.\mathrm{CDCl}_{3}\right): \delta 2.21\left(\mathrm{~s}, 3 \mathrm{H}, \mathrm{NCH}_{3}\right), 2.40-2.54(\mathrm{~m}, 8 \mathrm{H}$, piperazine $), 2.82-2.94\left(\mathrm{~m}, 2 \mathrm{H}, \mathrm{ArCH}_{2}\right), 3.19(\mathrm{~S}, 2 \mathrm{H}$, $\left.\mathrm{COCH}_{2} \mathrm{~N}\right)$ 3.38-3.49 (m, 2H, NCH$), 3.74(\mathrm{~s}, 3 \mathrm{H}, \mathrm{OMe})$, $3.84(\mathrm{~s}, 3 \mathrm{H}, \mathrm{OMe}), 6.54(\mathrm{~s}, 1 \mathrm{H}, \mathrm{CH}), 6.73(\mathrm{~s}, 1 \mathrm{H}, \mathrm{ArH})$, 6.84 (s, 1H, ArH), 7.40-7.46 (d, 2H, ArH), 8.14-8.19 (d, $2 \mathrm{H}, \mathrm{ArH})$. Mass (EI): m/z (\%): $\mathrm{M}^{+} 454$ (10.13), 313 (4.72), 267 (4.00), 113 (100). IR ( $\left.\mathrm{KBr}, \mathrm{cm}^{-1}\right): 1641$. 
1-(3,4-dihydro-6,7-dimethoxy-1-(4-nitrophenyl)isoquinolin-2(1H)-yl)-2-morpholinoethanone (13d)

Yield $55.0 \%$, Gummy material. ${ }^{1} \mathrm{H}$ NMR $(400 \mathrm{MHz}$, $\left.\mathrm{CDCl}_{3}\right): \delta 2.44-2.52\left(\mathrm{~m}, 4 \mathrm{H}, \mathrm{N}\left(\mathrm{CH}_{2}\right)_{2}\right), 2.77-2.83(\mathrm{~m}$, $\left.4 \mathrm{H}, \mathrm{O}\left(\mathrm{CH}_{2}\right)_{2}\right), 2.75-2.80\left(\mathrm{~m}, 2 \mathrm{H}, \mathrm{ArCH}_{2}\right), 3.33(\mathrm{~S}, 2 \mathrm{H}$, $\left.\mathrm{COCH}_{2} \mathrm{~N}\right), 3.54-3.64\left(\mathrm{~m}, 2 \mathrm{H}, \mathrm{NCH}_{2}\right), 3.74(\mathrm{~s}, 3 \mathrm{H}$, $\mathrm{OMe}), 3.80(\mathrm{~s}, 3 \mathrm{H}, \mathrm{OMe}), 6.20(\mathrm{~s}, 1 \mathrm{H}, \mathrm{CH}), 6.56(\mathrm{~s}, 1 \mathrm{H}$ ,ArH), 6.67 (s, 1H, ArH), 7.30-7.66 (m, 4H, ArH). Mass (EI): m/z (\%): $M^{+} 441$ (5.00), 309 (20.83), 100 (100). IR $\left(\mathrm{KBr}, \mathrm{cm}^{-1}\right): 1636$.

1-(1-(4-chlorophenyl)-3,4-dihydro-6,7-dimethoxyisoquinolin-2(1H)-yl)-2-(4-methylpiperazin-1yl)ethanone (13e)

Yield $58.9 \%$, Gummy material. ${ }^{1} \mathrm{H}$ NMR $(400 \mathrm{MHz}$, $\left.\mathrm{CDCl}_{3}\right): \delta 2.26\left(\mathrm{~s}, 3 \mathrm{H}, \mathrm{NCH}_{3}\right), 2.48-2.58(\mathrm{~m}, 8 \mathrm{H}$, piperazine $), 2.78-2.86\left(\mathrm{~m}, 2 \mathrm{H}, \mathrm{ArCH}_{2}\right), 3.28(\mathrm{~s}, 2 \mathrm{H}$, $\left.\mathrm{COCH}_{2} \mathrm{~N}\right), 3.42-3.50\left(\mathrm{~m}, 2 \mathrm{H}, \mathrm{NCH}_{2}\right), 3.71(\mathrm{~s}, 3 \mathrm{H}$, $\mathrm{OMe}$ ), 3.78 (s, 3H, OMe), $6.48(\mathrm{~s}, 1 \mathrm{H}, \mathrm{CH}), 6.66$ (s, 1H ,ArH), 6.78 (s, 1H, ArH), 7.22-7.40 (m, 4H, ArH). Mass (EI): $\mathrm{m} / \mathrm{z}(\%): \mathrm{M}^{+} 443$ (50), 342 (70), 302 (30), 113 (95), 70 (100). IR ( $\left.\mathrm{KBr}, \mathrm{cm}^{-1}\right): 1636$.

1-(1-(4-chlorophenyl)-3,4-dihydro-6,7-dimethoxyisoquinolin-2(1H)-yl)-2-morpholinoethanone (13f)

Yield $50.0 \%$, Gummy material. ${ }^{1} \mathrm{H}$ NMR $(400 \mathrm{MHz}$, $\left.\mathrm{CDCl}_{3}\right): \delta$ 2.32-2.42 (m, 4H, N( $\left.\left.\mathrm{CH}_{2}\right)_{2}\right), 2.68-2.74(\mathrm{~m}$, $\left.4 \mathrm{H}, \mathrm{O}\left(\mathrm{CH}_{2}\right)_{2}\right), 2.70-2.76\left(\mathrm{~m}, 2 \mathrm{H}, \mathrm{ArCH}_{2}\right), 3.26(\mathrm{~S}, 2 \mathrm{H}$, $\left.\mathrm{COCH}_{2} \mathrm{~N}\right), 3.62-3.66\left(\mathrm{~m}, 2 \mathrm{H}, \mathrm{NCH}_{2}\right), 3.73(\mathrm{~s}, 3 \mathrm{H}$, $\mathrm{OMe}), 3.78$ (s, 3H, OMe), $6.18(\mathrm{~s}, 1 \mathrm{H}, \mathrm{CH}), 6.54(\mathrm{~s}, 1 \mathrm{H}$ ,ArH), $6.64(\mathrm{~s}, 1 \mathrm{H}, \mathrm{ArH}), 7.34-7.64(\mathrm{~m}, 4 \mathrm{H}, \mathrm{ArH})$. Mass (EI): m/z (\%): $\mathrm{M}^{+} 430.5$ (7.37), 301.5 (5.47), 100 (100). IR $\left(\mathrm{KBr}, \mathrm{cm}^{-1}\right): 1642$.

Synthesis of N-(4-((E)-(3,4-dimethoxyphenethylimino)methyl)benzylidene)-2-(3,4-dimethoxyphenyl) ethanamine (15)

A mixture of 2-(3',4'-dimethoxyphenyl)ethylamine $(1.8 \mathrm{~g}, 10 \mathrm{mmol})$ and pthaldehyde $(6.9 \mathrm{mmol})$ in anhydrous toluene $(50 \mathrm{~mL})$ was refluxed for $3 \mathrm{~h}$ and then cooled and evaporated in vacuo. The oil residue was treated with diethyl ether to give a solid residue, which was crystallized from ethanol to afford the desired diimine.

Yield $55.9 \%$, White crystals. Melting point $113{ }^{\circ} \mathrm{C}$. ${ }^{1} \mathrm{H}$ NMR $\left(400 \mathrm{MHz}, \mathrm{CDCl}_{3}\right): \delta$ 2.98-3.02(t, $2 \mathrm{H} \mathrm{X} \mathrm{2,}$ $\left.\mathrm{ArCH}_{2}\right), 3.85-3.90\left(\mathrm{~m}, 2 \mathrm{H} \mathrm{X} \mathrm{2,} \mathrm{NCH}_{2}\right), 3.82,3.87(\mathrm{~s},(3 \mathrm{H}$ $X$ 2) $\left.X 2, \mathrm{OCH}_{3}\right), 6.75-6.82(\mathrm{~m}, 3 \mathrm{H} \times 2, \mathrm{ArH}), 7.7(\mathrm{~s}, 2 \mathrm{H}$ $\mathrm{X} 2, \mathrm{ArH}), 8.14\left(\mathrm{~s}, 1 \mathrm{H} \times 2\right.$, =CH). Mass (El): $\mathrm{m} / \mathrm{z}(\%): \mathrm{M}^{+}$ 460(3), 256(23.74), 185(15), 128(26.58), 55(100). IR $\left(\mathrm{KBr}, \mathrm{cm}^{-1}\right): 1641$.
Synthesis of 1,2,3,4-tetrahydro-1-(4-(1,2,3,4-tetrahydro-6,7-dimethoxyiso quinolin-1-yl)phenyl)-6,7dimethoxyisoquinoline (16)

Trifluoroacetic acid $(10 \mathrm{~mL})$ was added to a solution of compound $15(2.1 \mathrm{mmol})$, and the mixture was refluxed for $1.5 \mathrm{~h}$. The reaction was quenched by adding water, and the mixture was basified $(\mathrm{pH} 8-9)$ with sodium hydroxide to give the isoquinoline derivative as a solid. The crude product was collected by filtration and purified by crystallization with $\mathrm{MeOH}$ to afford compound.

Yield $46.9 \%$, White crystals. Melting point $224{ }^{\circ} \mathrm{C}$. ${ }^{1} \mathrm{H}$ NMR $\left(400 \mathrm{MHz}, \mathrm{CDCl}_{3}\right): \delta 1.76(\mathrm{~s}, 1 \mathrm{H} \times 2, \mathrm{NH})$, 2.75-3.23 (m, $\left.4 \mathrm{H} \mathrm{X} \mathrm{2,} \mathrm{NCH}_{2} \mathrm{CH}_{2}\right), 3.64,3.90(\mathrm{~s}, 6 \mathrm{H} \times 2$, $\left.\mathrm{OCH}_{3}\right), 5.06(\mathrm{~s}, 1 \mathrm{H} \mathrm{X} \mathrm{2,} \mathrm{CH}), 6.25(\mathrm{~s}, 1 \mathrm{H} \mathrm{X} \mathrm{2,} \mathrm{ArH),}$ 6.64 (s, $1 \mathrm{H} \times 2, \mathrm{ArH}), 7.19-7.28$ (d, $2 \mathrm{H} \times 2, \mathrm{ArH})$. Mass (EI): m/z (\%): $\mathrm{M}^{+} 460$ (3), 256 (21.07), 128 (28.03), 57 (100). IR ( $\left.\mathrm{KBr}, \mathrm{cm}^{-1}\right): 3374$.

\section{CONCLUSION}

A new approach for the preparation of $\mathrm{N}$-substituted THIQ derivatives has been reported. THIQ derivatives (13a-f) are synthesized by four step synthetic strategy via a Pictet-Spengler condensation. A new bisisoquinoline derivative, namely, 1,2,3,4-tetrahydro-1-(4(1,2,3,4-tetrahydro-6,7-dimethoxyisoquinolin-1-

yl)phenyl)-6,7-dimethoxyisoquinoline (16) has also been synthesized. The products and intermediates are characterized by IR, ${ }^{1} \mathrm{H}$ NMR and mass spectroscopy. The spectra of the synthesized compounds are matching with the desired products.

\section{ACKNOWLEDGEMENT}

We wish to thank Head, Department of Chemistry, The Maharaja Sayajirao University of Baroda for providing research facilities.

\section{REFERENCES}

[1] Locher C, Peerzada N Synthesis of some N-methyl-1,2,3,4tetrahydroisoquinolines by Friedel-Crafts cyclisation using benzotriazole auxiliary. Chem Soc Perkin Trans 1 1999; 17984

http://dx.doi.org/10.1039/a807543c

[2] He Y, Nikulin VI, Vansal SS, Feller DR, Miller DD. Synthesis and human $\beta$-adrenoceptor activity of 1-(3,5-diiodo-4methoxybenzyl)-1,2,3,4-tetrahydroisoquinolin-6-ol derivatives in vitro. J Med Chem 2000; 43: 591-8. http://dx.doi.org/10.1021/jm990463j

[3] Lees GJ. Pharmacology of AMPA/kainate receptor ligands and their therapeutic potential in neurological and psychiatric disorders. Drugs 2000; 59: 33-78.

http://dx.doi.org/10.2165/00003495-200059010-00004 
[4] Lin Z, Kabada PK. Molecular targets for the rational design of antiepileptic drugs and related neuroprotective agents. Med Res Rev 1997; 17: 537-72

http://dx.doi.org/10.1002/(SICI)1098-

1128(199711)17:6<537::AID-MED3>3.0.CO;2-2

[5] Rogawski MA, Donevan SD. AMPA receptors in epilepsy and as targets for antiepileptic drugs. Adv Neurol 1999; 79: 94763. http://www.ncbi.nlm.nih.gov/pubmed/10514878

[6] Gitto R, Barreca ML, De Luca L, et al. Discovery of a novel and highly potent noncompetitive AMPA receptor antagonist. J Med Chem 2003; 46: 197-00. http://dx.doi.org/10.1021/jm0210008

[7] Kaur J, Ghosh NN, Chandra R. Synthesis and antispasmodic activity evaluation of bis-(papaverine) analogues. Chem Pharm Bull 2004; 52: 316-21. http://dx.doi.org/10.1248/cpb.52.316

[8] Chandra R, Kaur J, Talwar A, Ghosh NN. Synthesis and antispasmodic effect of aryl substituted N-carbamoyl/ thiocarbamoyl isoquinolines. Arkivoc 2001; (viii): 129-35. http://dx.doi.org/10.3998/ark.5550190.0002.814

[9] Kaur J, Ghosh NN, Talwar A, Chandra R. Synthesis of $\mathrm{N}$ Substituted Piperazinyl Carbamoyl and Acetyl Derivatives of Tetrahydropapaverine: Potent Antispasmodic Agents. Chem Pharm Bull 2002; 50: 1223-8. http://dx.doi.org/10.1248/cpb.50.1223

[10] Kitamura $M$, Hsiao $Y$, Otha $M$, et al. General asymmetric synthesis of isoquinoline alkaloids. Enantioselective hydrogenation of enamides catalyzed by BINAPruthenium(I1) complexes. J Org Chem 1994; 59: 297-310. http://dx.doi.org/10.1021/jo00081a007

[11] Scott JD, Williams RM. Chemistry and biology of the tetrahydroisoquinoline antitumor antibiotics. Chem Rev 2002; 102: 1669-730.

http://dx.doi.org/10.1021/cr010212u

[12] Iwasa $K$, Moriyasu M, Tachibana $Y$, et al. Simple isoquinoline and benzylisoquinoline alkaloids as potential antimicrobial, antimalarial, cytotoxic, and anti-HIV agents. Bioorg Med Chem 2001; 9: 2871-4.

http://dx.doi.org/10.1016/S0968-0896(01)00154-7

[13] Minor DL, Wyrick SD, Charifson PS, Watts VJ, Nichols DE, Mailman RB. Synthesis and molecular modeling of 1-phenylI,2,3,4-tetrahydroisoquinolines and related 5,6,8,9-tetrahydro$13 \mathrm{bH}$-dibenzo[a,h]quinolizineass D1 dopamine antagonists. J Med Chem 1994; 37: 4317-28. http://dx.doi.org/10.1021/jm00051a008

[14] Tan GT, Pezzuto JM, Kinghorn AD, Hughes SH. Evaluation of natural products as inhibitors of human immunodeficiency virus type 1 (HIV-1) reverse transcriptase ${ }^{1}$. J Nat Prod 1991; 54: 143-54.

http://dx.doi.org/10.1021/np50073a012

[15] Pham VC, Ma J, Thomas SJ, Xu Z, Hecht SM. Alkaloids from alangium javanicum and alangium grisolleoides that mediate $\mathrm{Cu}^{2+}$-dependent DNA strand scission. J Nat Prod 2005; 68: 1147-52.

http://dx.doi.org/10.1021/np058013j

[16] Oku N, Matsunaga S, van Soest RWM, Fusetani N Renieramycin J, a highly cytotoxic tetrahydroisoquinoline alkaloid, from a marine sponge Neopetrosia $\mathrm{sp}^{1}$. J Nat Prod 2003; 66: 1136-9.

http://dx.doi.org/10.1021/np030092g

[17] Naito R, Yonetoku Y, Okamoto Y, Toyoshima A, Ikeda K Takeuchi $M$. Synthesis and antimuscarinic properties of quinuclidin-3-yl 1,2,3,4-tetrahydroisoquinoline-2-carboxylate derivatives as novel muscarinic receptor antagonists ${ }^{1}$. J Med Chem 2005; 48: 6597-606.

http://dx.doi.org/10.1021/jm050099q

[18] Castro L, Ball RG, Broughton HB, et al. Controlled modification of acidity in cholecystokinin $B$ receptor antagonists: $\quad \mathrm{N}$-(1,4-Benzodiazepin-3-yl)-N'-[3-(tetrazol-5ylamino)phenyl]ureas. J Med Chem 1996; 39: 842-9. http://dx.doi.org/10.1021/jm9506736

[19] Nakamura S, Tanaka M, Taniguchi T, Uchiyama M, Ohwada T. Stereoselectivity of superacid-catalyzed pictet-spengler cyclization reactions. Org Lett 2003; 5: 2087-90. http://dx.doi.org/10.1021/ol034526a

[20] Cox ED, Cook JM. The Pictet-Spengler condensation: a new direction for an old reaction. Chem Rev 1995; 95: 1797-842. http://dx.doi.org/10.1021/cr00038a004

[21] Semple G, Ryder H, Rooker DP, et al. (3R)- N-(1-(tertButylcarbonylmethyl)-2,3-dihydro-2-oxo-5-(2-pyridyl)-1H-1,4benzodiazepin-3-yl)- $N$ '-(3-(methylamino)phenyl)urea (YF476): A potent and orally active gastrin/CCK-B antagonist. J Med Chem 1997; 40: 331-41. http://dx.doi.org/10.1021/jm960669+

[22] March J. Advanced Organic Chemistry: Reactions, Mechanisms and Structure. New York: John Wiley \& Sons, 4th ed 1992.

[23] Larghi EL, Amongero M, Bracca ABJ, Kaufman TS. The intermolecular Pictet-Spengler condensation with chiral carbonyl derivatives in the stereoselective syntheses of optically active isoquinoline and indole alkaloids. Arkivoc 2005; (vii): 98-153.

http://dx.doi.org/10.3998/ark.5550190.0006.c09 